\title{
Adsorption and desorption characteristics of polyphenols from Eucommia ulmoides Oliv. leaves with macroporous resin and its inhibitory effect on $\alpha$-amylase and $\alpha$-glucosidase
}

\author{
Zhihong Wang ${ }^{1,3}$, Sheng Peng ${ }^{2}$, Mijun Peng ${ }^{1}$, Zhigang She ${ }^{3}$, Qiuling Yang ${ }^{1}$, Tao Huang ${ }^{1}$ \\ ${ }^{1}$ Guangdong Provincial Key Laboratory of Emergency Test for Dangerous Chemicals, Guangdong Institute of Analysis (China National Analytical \\ Center Guangzhou), Guangdong Academy of Sciences, Guangzhou, China; ${ }^{2}$ Key Laboratory of Hunan Forest Products and Chemical Industry \\ Engineering, Jishou University, Zhangjiajie, China; ${ }^{3}$ School of Chemistry, Sun Yat-Sen University, Guangzhou, China \\ Contributions: (I) Conception and design: Z Wang, S Peng, M Peng, Z She; (II) Administrative support: Z Wang, S Peng, M Peng; (III) Provision of \\ study materials or patients: Z Wang, S Peng; (IV) Collection and assembly of data: S Peng, Q Yang; (V) Data analysis and interpretation: Z Wang, T \\ Huang; (VI) Manuscript writing: All authors; (VII) Final approval of manuscript: All authors. \\ Correspondence to: Prof. Mijun Peng. Building 34, No.100 Xianlie Zhong Road, Yuexiu District, Guangzhou, China. Email: pengmj163@163.com; \\ Prof. Zhigang She. No. 135, Xingang Xi Road, Guangzhou, China. Email: cesshzhg@mail.sysu.edu.cn.
}

Background: Eucommia ulmoides Oliv. (EUO) was a traditional Chinese herb, its leaves were abundant in China, and polyphenol compounds were considered to be an important active ingredient in Eucommia ulmoides Oliv. leaves (EUOL). However, previous research mainly focused on compound identification and extraction process, there were few reported on the efficient enrichment process and biological activity evaluation of polyphenols in EUOL.

Methods: The adsorption and desorption characteristics of twelve different resins (HPD-100, HPD-300, HPD-600, D-3250, X-5, D-140, NKA-9, NKA-II, D-101, AB-8, S-8 and Polyamide) were investigated to develop an efficient method for the enrichment of polyphenol from EUOL, and the static adsorption, kinetics, isotherm and thermodynamics of the polyphenol from EUOL were analyzed. The eluted component was obtained through dynamic elution, and its main polyphenol compounds were detected by high-phase liquid chromatography (HPLC) and the inhibitory effects on the enzyme activity of $\alpha$-amylase and $\alpha$-glucosidase was also evaluated for different elution components. Meanwhile, the binding of main polyphenol compounds to enzyme was also evaluated.

Results: The selected resins (HPD-300, HPD-600, D-3250, X-5, D-140, NKA-9, D-101 and AB-8) showed adsorption patterns that fitted well to the pseudo second-order kinetics. The intra-particle diffusion model demonstrated that the diffusion of polyphenol compounds on these resins were divided into three processes. For HPD-300, HPD-600 and NKA-9, the Freundlich model better described the adsorption isotherm behavior than the Langmuir model, and the adsorption of polyphenol was a physical, exothermic, and spontaneous process. Subsequently, dynamic elution was performed yielding a higher polyphenol content in a $60 \%$ ethanolwater elution component, and it also exhibited a higher inhibitory effect on $\alpha$-amylase and $\alpha$-glucosidase activity. Furthermore, as the main polyphenol compounds, chlorogenic acid, rutin, quercetin and kaempferol were used to simulate the binding to the enzyme protein through molecular docking technology. The results showed that quercetin had a higher docking score for $\alpha$-amylase, while rutin displayed superior binding to $\alpha$-glucosidase.

Conclusions: Therefore, polyphenols of EUOL could be enriched through macroporous resins and have the potential to be effective enzyme inhibitor.

Keywords: Eucommia ulmoides Oliv. leaves (EUOL); marcoporous resin; adsorption; desorption; polyphenol; inhibitory effect

Submitted Jun 20, 2020. Accepted for publication Aug 07, 2020.

doi: 10.21037/atm-20-5468

View this article at: http://dx.doi.org/10.21037/atm-20-5468

(c) Annals of Translational Medicine. All rights reserved. 


\section{Introduction}

Eucommia ulmoides Oliv. (EUO) is widely used as a native traditional medicinal plant, found to be abundant in China, and its bark, leaves, seeds and flowers can be applied as the feedstock for the production of bioactive substances (1). Previously, only Eucommia ulmoides Oliv. bark (EUOB) was used as medicinal materials. However, numerous research has indicated that Eucommia ulmoides Oliv. leaves (EUOL) are rich in natural active ingredients. Moreover, many phytochemical components identified in the bark is also found in the leaves $(2,3)$, and a total of 138 kinds of chemical compounds were separated, purified and identified from EUO bark and leaves. Meanwhile, EUOL has attracted widespread attention due to its abundance and availability, easy collection and rapid growth cycle. Furthermore, EUOL has been recorded officially in Chinese Pharmacopoeia since 2005 (4). Recently, EUOL has been regarded as a homologue substance of medicine and food by National Health Commission of the People's Republic of China, highlighting their potential in development value in the food and pharmaceutical industries. Because EUOL contain many natural active ingredients, such as polyphenols, lignans, iridoids and flavonoids, and their biological activity has excellent efficacy in treating diseases and imporving the quality of life. Traditionally, the comprehensive utilization rate of EUO is still very low. Only the bark is used as a medicinal material, and its chemical composition and pharmacological research are relatively extensive. In the past few decades, even in Japan and Korea, EUOL were used in food, but they were not fully utilized (1). Therefore, based on the advantages of EUOL, it has broad application prospects in pharmacological research and food development (tae, wine and nutritional supplement).

EUOL is an important raw material for food and drug development. Its phytochemical composition, biological activity and pharmacological research have continued to arouse substantial great interest over the past decades $(5,6)$, with intense focus on the efficient preparation of extracts, active compounds and analysis of biological activity. EUOB has many pharmacological actions such as anti-oxidation (7), antifungal (8), anti-inflammatory, antihypertensive (9), inhibiting adipogenic differentiation (10) and low-density lipoprotein oxidative modification (11). Due to the difficulties in obtaining EUOB, its leaves, which contain comparatively equivalent components as the bark (3), have been the preferred source in medical research. Similar to
EUOBs, EUOL also have a series of pharmacological effects including anti-oxidation (7), anti-aging, antimutagenicity (12), antihypertensive (9), hypoglycemic $(13,14)$, hypolipidemic (15) and preventing gastric mucosal injury (16). Therefore, the extraction, enrichment, separation and identification of bioactive ingredients in EUOL have been widely concerned, especially polyphenols, flavonoids, iridoids and lignans $(1,2,5)$.

The known benefits of EUOL are mainly derived from its rich natural active ingredients. It is worth noting that polyphenols are the main type of compounds in EUOL (5). At present, much focus on polyphenol research pertains to its effect in delaying the onset of diabetes $(17,18)$. Inhibitors of $\alpha$-amylase and $\alpha$-glucosidase activity are also often considered as potential active ingredients that delay type 2 diabetes $(19,20)$. Meanwhile, there were few studies on the systematic study of the enrichment process and biological activities of polyphenols in EUOL. To obtain the desired target compound, macroporous resins have been considered as an effective means for enrichment and purification of active ingredients from complex extracts. Traditional purification techniques, such as liquid-liquid extraction and silica gel column chromatography, have many deficiencies, such as high solvent consumption, residual organic solvents, and environmental pollution $(21,22)$. The advantages of using macroporous resins include easy operation, strong adsorption capacity, low running cost, low solvent consumption, product safety, and ease of resin regeneration. It has been successfully applied in the separation and enrichment of biologically active compounds from many natural products $(23,24)$. However, there are few reports on the systematic investigation of EUOL polyphenol adsorption and desorption characteristics using macroporous adsorption resin. Consequently, macroporous resins were the preferred material to develop a simple and efficient process for the preliminary enrichment and separation of polyphenol from EUOL.

The aim of this study was to investigate the adsorption and desorption behaviors of macroporous resins, namely HPD-100, HPD-300, HPD-600, D-3250, X-5, D-140, NKA-9, NKA-II, D-101, AB-8, S-8 and polyamide. Meanwhile, the static adsorption, kinetics, isotherm and thermodynamics of the polyphenol from EUOL were investigated. The target enrichment component was obtained from resins selected by gradient elution with different ratios of ethanol:water solution. The inhibitory effects of the eluates on $\alpha$-amylase and $\alpha$-glucosidase enzyme activity were evaluated, and the optimal active fraction of the enzyme inhibitor was screened by $\mathrm{IC}_{50}$ value, meanwhile, the chemical composition of the active site 
Table 1 Physical characteristics of the selected macroporous resins

\begin{tabular}{|c|c|c|c|c|c|}
\hline Resins & Polarity & Average pore diameter (nm) & Particle size $(\mathrm{mm})$ & Specific surface area $\left(\mathrm{m}^{2} / \mathrm{g}\right)$ & Pore volume $(\mathrm{mL} / \mathrm{g})$ \\
\hline HPD-300 & Nonpolar & $5.0-5.5$ & $0.3-1.2$ & $800-870$ & - \\
\hline HPD-600 & Polar & 8.0 & $0.3-1.2$ & $550-600$ & - \\
\hline D-3520 & Nonpolar & $8.5-9.0$ & $0.3-1.25$ & $480-520$ & $2.10-2.15$ \\
\hline D-140 & Nonpolar & 9.5 & - & $500-600$ & $1.00-1.50$ \\
\hline NKA-9 & Polar & $15.5-16.5$ & $0.315-1.25$ & $170-250$ & $1.00-1.04$ \\
\hline NKA-II & Polar & $14-16$ & $0.3-1.25$ & $950-1,250$ & $0.62-0.66$ \\
\hline D-101 & Nonpolar & $9-11$ & $0.3-1.25$ & $550-600$ & $1.50-1.70$ \\
\hline Polyamide & Polar & - & - & $5-10$ & - \\
\hline
\end{tabular}

was analyzed by high performance liquid chromatography (HPLC). Finally, molecular docking technology was used to evaluate the interaction strength between the polyphenol compounds and protein. This work would contribute to the understanding of polyphenol isolation and preparation from EUOL. Additionally, these findings have important reference value for its application to nutritional and pharmaceutical therapeutic development.

We present the following article in accordance with the MDAR reporting checklist (available at http://dx.doi. org/10.21037/atm-20-5468).

\section{Methods}

\section{Materials}

EOULs were collected from the Cili Du-zhong Forestry Centre (Zhangjiajie, China). Fresh leaves were dehydration dried at $60{ }^{\circ} \mathrm{C}$ by electro-thermostatic blast oven (Shanghai Jing Hong Laboratory Instrument Co., Ltd. Shanghai, China), and pulverized before storage at $4{ }^{\circ} \mathrm{C}$. Chromatographic grade methanol was provided by Merch (Darmstadt, Germany). Deionized water was purified using a Merck Millipore Synergy UV water purification system (Billerica, MA, USA). All other chemicals used were of analytical grade. Macroporous resins (HPD-100, HPD300, HPD-600, D-3250, X-5, D-140, NKA-9, NKAII, D-101, AB-8, S-8 and Polyamide) were supplied by Zhengzhou Qinshi Technology Co., Ltd. (Zhengzhou,
China). The chemical and physical properties of the tested resins were provided by the manufacturer and shown in the Table 1. Macropous resins were pretreated according to the manufacturer's recommendation. Prior to use, the resins were soaked with ethanol for $24 \mathrm{~h}$ and washed several times with deionized water. Subsequently, the resins were soaked in $1.0 \mathrm{M} \mathrm{NaOH}$ for $4 \mathrm{~h}$, then washed before undergoing another soak in $1.0 \mathrm{M} \mathrm{HCl}$ for $4 \mathrm{~h}$. Finally, the resins were thoroughly washed with deionized water before use.

\section{Extract methods}

The crude extract of EUOL was prepared through ultrasound-assisted extraction (UAE) technology. Briefly, $1.0 \mathrm{~kg}$ of dried EUOLs were ground into fine power using a laboratory mill (FW100, Taisite Instrument Co., Ltd., Tianjin, China) and subsequently mixed with 15 L $60 \%$ ethanol before UAE extraction for $30 \mathrm{~min}$. The extraction solution was collected by filtration. The residual paste underwent further extraction twice with the same process, using equal volumes of ethanol. The filtrates were combined and concentrated under a vacuum rotary evaporator. Subsequently, EOUL extract was obtained by freeze drying and stored at $-4^{\circ} \mathrm{C}$ before use.

\section{Static adsorption and desorption experiments}

The static adsorption and desorption characteristics on the 
selected resins were performed as follows: $1.0 \mathrm{~g}$ of pretreated macroporous resin (equal to $0.50 \mathrm{~g}$ dry resin) was placed with $50 \mathrm{~mL}$ of $10.0 \mathrm{mg} / \mathrm{mL}$ extracts solution in a $250 \mathrm{~mL}$ conical flask. Target compounds of the EUOL extract was analyzed before adsorption. The sealed flasks were shaken at $25^{\circ} \mathrm{C}$ for $24 \mathrm{~h}$ at $120 \mathrm{rpm}$. After adsorption, the absorbance of total polyphenols in the sample solution was detected. The adsorption capacity and ratio were calculated using the following equations $(25,26)$ :

Adsorption capacity:

$$
Q_{t}=\frac{\left(C_{0}-C_{t}\right) V_{i}}{W}
$$

Adsorption ration:

$$
E(\%)=\frac{C_{0}-C_{t}}{C_{0}} \times 100 \%
$$

After the adsorption equilibrium was achieved, the resins were washed with $50 \mathrm{~mL}$ distilled water. The wash procedure was repeated twice before discarding the

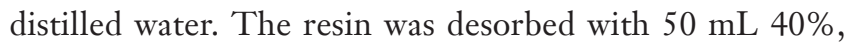
$70 \%$ and $100 \%(\mathrm{v} / \mathrm{v})$ ethanol solution, respectively, using a thermostatic oscillator at $25^{\circ} \mathrm{C}(120 \mathrm{rpm})$. A circulating water bath with a thermostat was applied to maintain the temperature within $\pm 0.1{ }^{\circ} \mathrm{C}$. The concentration of polyphenols in the desorption solutions were determined after $24 \mathrm{~h}$. The desorption ratio was calculated according to the following formula:

Desorption ration:

$$
D(\%)=\frac{C_{d} V_{d}}{\left(C_{0}-C_{e}\right) V_{i}}
$$

Where $\mathrm{Q}_{\mathrm{t}}$ is the adsorption capacity at adsorption equilibrium ( $\mathrm{mg} / \mathrm{g}$ dry resin), $\mathrm{C}_{0}$ is the initial concentration of crude extract solutes $(\mathrm{mg} / \mathrm{mL}), \mathrm{C}_{\mathrm{e}}$ is the concentration of equilibrium solutes $(\mathrm{mg} / \mathrm{mL}), \mathrm{C}_{\mathrm{d}}$ is the concentration of target compounds in the desorption solution $(\mathrm{mg} / \mathrm{mL}), \mathrm{V}_{\mathrm{i}}$ is the volume of the initial extraction solution $(\mathrm{mL})$, and $\mathrm{W}$ is the weight of the dry macroporous resin $(\mathrm{g})$, and $\mathrm{V}_{\mathrm{d}}$ is the volume of the desorption $(\mathrm{mL})$.

\section{Adsorption and desorption kinetics}

The hydrated resin (equal to $5.00 \mathrm{~g}$ dry resin) were mixed with $20 \mathrm{~mL}$ of extracts solution from EUOLs. Adsorption was performed on a thermostatic oscillator $(120 \mathrm{rpm})$ at $25^{\circ} \mathrm{C}$, where $1.0 \mathrm{~mL}$ of each solution was withdrawn at the time points of $0,5,15,35,55,85,115,145$ and $175 \mathrm{~min}$. The total target compounds were subsequently measured. Meanwhile, the kinetic behavior was evaluated.

The pseudo-first-order kinetic model was presented as:

$$
\ln \left(Q_{e}-Q_{t}\right)=-k_{1} t+\ln Q_{e}
$$

The pseudo-second-order kinetic model was shown as:

$$
\frac{1}{Q_{t}}=\frac{1}{k_{2} Q_{e}^{2}} \cdot \frac{1}{t}+\frac{1}{Q_{e}}
$$

The particle diffusion kinetic model was expressed as:

$$
Q_{t}=k_{d} \cdot t^{1 / 2}+C
$$

In the adsorption kinetic and thermodynamics model equations, $\mathrm{k}_{1}, \mathrm{k}_{2}$ and $\mathrm{k}_{\mathrm{d}}$ refer to the rate constants of pseudofirst-order, pseudo-second-order, and particle diffusion kinetic models in the adsorption process, respectively. $\mathrm{C}$ is the constant in the particle diffusion kinetic model (27).

In kinetic experiments, after adsorption equilibrium, these resins were washed twice through distilled water. Sixty percent ethanol solution was used as a desorption solvent. The total polyphenol concentration in the sample was detected at the time points of $0,5,15,25,45,65,95,125$ and $155 \mathrm{~min}$. The desorption curves were obtained, based on the concentration in the solvent.

\section{Adsorption isotherms and thermodynamics of the selected resins}

The adsorption isotherm was a function of the equilibrium concentration of the adsorbed substance and the adsorbent content in a solution at a specific temperature. The selected resins were added to stoppered flasks containing $10 \mathrm{~mL}$ polyphenol solutions of different concentrations. Adsorption was conducted at three different temperatures $\left(25,35\right.$ and $\left.45^{\circ} \mathrm{C}\right)$ in a $25 \mathrm{~mL}$ flask with a stopper. The adsorption isotherms for total polyphenols were determined using Langmuir and Freundlich equation (28).

The Langmuir equation and its variable form:

$$
\begin{aligned}
& Q_{e}=\frac{q_{m} K_{L} C_{e}}{1+K_{L} C_{e}} \\
& \frac{C_{e}}{Q_{e}}=\frac{1}{Q_{m}} \cdot C_{e}+\frac{1}{q_{m} K_{L}}
\end{aligned}
$$

The Freundlich equation and its variable form: 


$$
\begin{aligned}
& Q_{e}=K_{F} C_{e}^{1 / n} \\
& \ln Q_{e}=\frac{1}{n} \cdot \ln C_{e}+\ln K_{F}
\end{aligned}
$$

Where $K_{\mathrm{L}}$ is the affinity parameter between resin and target compounds. $K_{\mathrm{F}}$ is the adsorption capacity of the resin.

Adsorption thermodynamics is necessary to determine the mechanism involved in the adsorption process and the inherent energy change of the resin after adsorption (29). The Gibbs free energy change $(\Delta \mathrm{G})$, enthalpy change $(\Delta \mathrm{H})$ and entropy change $(\Delta S)$ were evaluated to describe the total flavonoids adsorption thermodynamics at different temperatures. Enthalpy changes $(\Delta \mathrm{H})$ were calculated by the Van't Hoff equation:

$$
\begin{aligned}
& \ln K=-\frac{\Delta H}{R T}+\frac{\Delta S}{R} \\
& \Delta S=\frac{\Delta H-\Delta G}{T}
\end{aligned}
$$

Where $1 / \mathrm{n}$ was the adsorption intensity of the resin. $\mathrm{R}$ is the universal gas constant $[8.314 \mathrm{~J} /(\mathrm{mol} \mathrm{K})]$. T denotes the absolute temperature $(\mathrm{K})$ and A refers to a constant. $\mathrm{K}$ is thermodynamic equilibrium constant, which is determined by plotting $\ln \left(\mathrm{Q}_{\mathrm{e}} / \mathrm{C}_{\mathrm{e}}\right)$ versus $\mathrm{Q}_{\mathrm{e}}$ and extrapolating to zero $\mathrm{Q}_{\mathrm{e}}(30)$.

\section{Enrichment of target compounds by resin column}

Dynamic adsorption and desorption experiments were performed in glass column (length $30 \mathrm{~cm} \times \Phi 2.5 \mathrm{~cm}$ ), wetpacked with the selected resin $\left(150 \mathrm{~mL}, 25^{\circ} \mathrm{C}\right)$. The aqueous extract solutions $(10.0 \mathrm{mg} / \mathrm{mL})$ were adsorbed and then eluted by increasing concentrations of ethanol $(0,20 \%$, $40 \%, 60 \%, 80 \%$ and $100 \%$ ). The volume of each solution was 3.0 BV. Eluates were collected and concentrated under reduced pressure in a rotary evaporator, and the total polyphenol content in the samples were detected by colorimetry. Additional target components in the sample were simultaneously detected by HPLC.

\section{Determination of total phenolic content}

Total phenolic content was determined using the method of Wang [2019] (31), with some modifications. Two hundred microliters of each sample were transferred to a $10 \mathrm{~mL}$ of volumetric flask, to which $0.5 \mathrm{~mL}$ of undiluted FolinCiocalteu reagent was added. After $1 \mathrm{~min}, 1.5 \mathrm{~mL}$ of $15 \%$ (w/v) $\mathrm{Na}_{2} \mathrm{CO}_{3}$ was added and the volume was made up to $10 \mathrm{~mL}$ with $\mathrm{H}_{2} \mathrm{O}$. After $60 \mathrm{~min}$ of incubation at $25^{\circ} \mathrm{C}$, the absorbance was measured at $760 \mathrm{~nm}$. Total phenolic content was calculated on the basis of the standard curve for gallic acid and expressed as $\mathrm{mg}$ of gallic acid equivalents $\mathrm{g}$ of sample. The calibration curve $\mathrm{Y}=0.0912 \mathrm{X}+0.0455$ (six data points) was linear with $\mathrm{R}^{2}=0.9995$.

\section{HPLC analysis}

Based on previous reports (32), the concentrations of compounds determined by HPLC with UV/Vis detection, employing a SHIMADZU HPLC system (Japan) contained a Smart line DGU-20A online degasser, a Smartline LC$20 \mathrm{AB}$ quaternary pump, and the diode array detection. The analytical column was an ODS C18. The injection volume was $10 \mu \mathrm{L}$ for each analysis. The temperature of the auto-sampler (SIL-20AC) was maintained at $30{ }^{\circ} \mathrm{C}$. Chromatograms were run at $0.8 \mathrm{~mL} / \mathrm{min}$ with isocratic elution. Gradient elution was performed as follows: 0.00-6.00 $\mathrm{min}, 6.0 \% \mathrm{~A}, 94 \% \mathrm{~B}$; 6.00-13.00 $\mathrm{min}, 6.0-13 \%$ A, $94-87 \%$ B; $13.00-14.50 \mathrm{~min}, 13-18 \% \mathrm{~A}, 87-82 \% \mathrm{~B}$; 14.50-22.00 min, 18-20\% A, 82-80\% B; 22.00-30.00 min, 20-40\% A, 80-60\% B; 30.00-32.00 min, 40-45\% A, 60$55 \% \mathrm{~B} ; 32.00-45.00 \mathrm{~min}, 45 \% \mathrm{~A}, 55 \% \mathrm{~B} ; 45.00-46.00 \mathrm{~min}$, 45-63\% A, 55-37\% B; 46.00-66.00 min, 63\% A, 37\% B; 66.00-68.00 min, 63-30\% A, 37-70\% B; 68.00-70.00 min, 30-6.0\% A, 70-94\% B; 70.00-90.00 min, 6.0\% A, 94\% B.

\section{Enzyme inbibition activity assay}

The $\alpha$-glucosidase inhibition activity assay was conducted based on the method described from previous literature reports with some modifications $(33,34)$. $\alpha$-Glucosidase solution $(0.5 \mathrm{mg} / \mathrm{mL})$ was prepared in phosphate buffer and stored at low temperature before use. Briefly, $200 \mu \mathrm{L}$ of $0.5 \mathrm{mg} / \mathrm{mL} \alpha$-glucosidase solution and $150 \mu \mathrm{L}$ of target compound solutions at various concentrations were added to each tube containing $2.0 \mathrm{~mL}$ phosphate buffer. Before the p-nitrophenyl- $\alpha-\mathrm{D}$-glucopyranoside (PNPG) solution $(150 \mu \mathrm{L}$ of $5.0 \mathrm{mmol} / \mathrm{L})$ was added to initiate reaction, the mixture system was incubated at $37{ }^{\circ} \mathrm{C}$ for $10 \mathrm{~min}$. Samples were again subjected to incubation at $37^{\circ} \mathrm{C}$ for another $10 \mathrm{~min}$, before termination by the addition of $2.5 \mathrm{~mL}$ of $\mathrm{Na}_{2} \mathrm{CO}_{3}(0.1 \mathrm{~mol} / \mathrm{L})$. The absorbance of each tube was measured at $405 \mathrm{~nm}$ by a UV-visible spectrophotometer. For this test, acarbose was used as a positive control.

The $\alpha$-amylase inhibition activity assay was performed, 


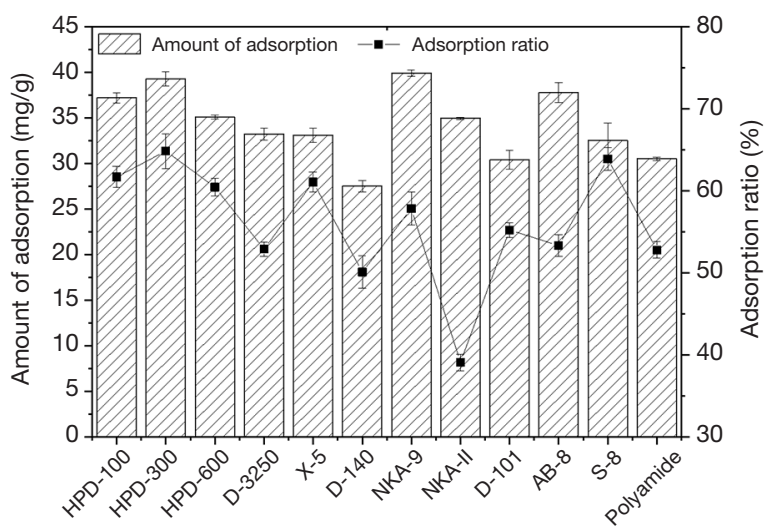

Figure 1 Adsorption capacity and adsorption ratio of different resins.

using a method previously reported with a slight modification $(33,34)$. The substrate solution was prepared by heating starch in phosphate buffer at $100{ }^{\circ} \mathrm{C}$, until transparent. The $\alpha$-amylase solution was also prepared using phosphate buffer ( $\mathrm{pH}$ 6.9) before the experiment. Briefly, the reaction system was composed of $\alpha$-amylase solution and sample solution at different concentrations. Following preincubation at $37^{\circ} \mathrm{C}$ for $10 \mathrm{~min}, 300 \mu \mathrm{L}$ of the substrate was pipetted into each tube to start the reaction. Subsequently, the reaction was undertaken at $37^{\circ} \mathrm{C}$ for $10 \mathrm{~min}$ before termination with $1.0 \mathrm{~mL}$ of $96 \mathrm{mM}$ 3,5-dinitrosalicylic acid (DNS) colour reagent. All samples were placed in boiling water for $5.0 \mathrm{~min}$ and cooled to room temperature. The final volume of reaction solution was made up to $10 \mathrm{~mL}$ using distilled water, and the absorbance was recorded at $540 \mathrm{~nm}$ through the UV-visible spectrophotometer. Acarbose was used as a positive control. The value of $\mathrm{IC}_{50}$ was calculated to evaluate the effect of enzyme inhibitors.

\section{Docking analysis}

Docking studies were performed with SYBYL X 2.0 software. The $\alpha$-glucosidase (2ZE0) and $\alpha$-amylase (1CLV) structures were obtained from the Protein Data Bank (PDB), and the polyphenol three dimensional structures were generated from PM3 semi-empirical calculations using Chem3D Ultra 14.0. The docking tool was used to remove solvent water molecules and adjusted atoms and charges, based on the three-dimensional structures of the proteins. The entire protein was selected for potential binding sites. The docking runs on the SYBYL X 2.0 docking engine with regular accuracy and 100 candidate poses. The binding of various compounds to proteins were evaluated upon completion of docking. The degree of binding was based on the score, with data from the enzyme inhibition test used for comprehensive analysis.

\section{Statistical analysis}

All the tests were carried out in the triplicate and the results were expressed as mean \pm standard deviation.

\section{Results}

\section{Static adsorption and desorption capacities}

The adsorption and desorption capabilities of the resin depend on the target compound and the adsorbent. Different resins exhibit varying polarity, particle size, specific surface area, pore diameter and chemical structure (35). The target compound, adsorbent and solvent interact when a solute undergoes the adsorption process (36). The adsorption of phenolic compounds on macroporous resin is via physical mechanisms through van der Waals force, or hydrogen bonding. Additionally, the $\pi-\pi$ conjugation between phenolics and benzene rings of resins cannot be overlooked (37). Polyphenols containing benzene rings and hydrogen groups may be non-polar or polar (38). Therefore, twelve resins including HPD-100, HPD-300, HPD-600, D-3250, X-5, D-140, NKA-9, NKA-II, D-101, AB-8, S-8 and polyamide, with surface area and average pore diameter ranging from non-polarity to polarity, were screened to adsorb and desorb phenolic compounds from EUOL. The parameters of resins are shown in Table 1.

The static adsorption capacity and adsorption rate of polyphenols from EUOL on twelve macroporous resins were initially investigated, with the results displayed in the Figure 1. The HPD-300 and NKA-9 resins showed high adsorption capacity of 39.29 and $39.91 \mathrm{mg} / \mathrm{g}$, respectively. This was slightly higher than that of HPD100 and AB-8 (37.18 and $37.78 \mathrm{mg} / \mathrm{g}$ ), while D-140 showed poorer performance compared to other resins. Among macroporous adsorption resins, non-polar type D-3250, $\mathrm{X}-5$ and S-8 displayed similar adsorption capacity (33.20, 33.07 and $32.50 \mathrm{mg} / \mathrm{g})$. The polar type HPD-600 and NKA-II (35.10 and $34.94 \mathrm{mg} / \mathrm{g}$ ) displayed slightly higher adsorption capacity than these three resins. However, the adsorption capacity of polyamide and D-101 were 30.50 and $30.39 \mathrm{mg} / \mathrm{g}$, respectively, they were only displayed 


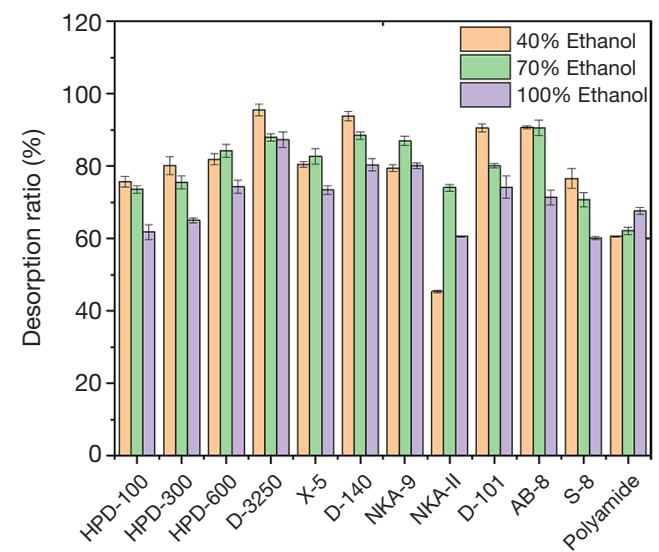

Figure 2 Desorption ratio of different resins with varying ethanol concentrations.

superiority to D-140 $(27.50 \mathrm{mg} / \mathrm{g})$. It is worth noting that the adsorption rate and resin amount exhibited the same trend. The adsorption ratios of polyphenol for all resins used in this study decreased in the order of NKA-9 $>$ HPD-300 > AB-8 > HPD-100 > NKA-II > HPD-600 > D-3250 $>$ S-8 $>$ polymide $>$ D-101 $>$ D-140. Based on the physical properties of the resins, the results suggest that macroporous cation resin is superior to that of microporousanion and adsorption resins. The adsorption of phenolic compounds mainly depends on the chemical and physical properties of the resin, including interaction forces, surface polarity, particle size and surface area (26). Non-polar type HPD-300 has the highest specific surface area compared to other adsorption resins, which is consistent with our results. Additionally, van der Waals forces and hydrogen bonds are the common mechanisms in the adsorption process $(25,26)$. Resins of varying characteristics exhibit different effects under the same conditions. The adsorption and desorption of resins to polyphenols from EUOL were affected by many factors, such as the polarity and specific surface area of resin, solution properties. For example, polyamide resin is a common adsorbent widely used in the separation of effective components of plants or Chinese medicines, especially for the enrichment of polyphenols and flavonoids (39). However, due to the composition of the raw materials, the actual adsorption capacity is often affected by the physical and chemical properties of the other components.

The desorption ratio of EUOL phenolics on all selected resins, using different desorption solvents $(40 \%, 70 \%$ and
$100 \%$ ethanol solution) are shown in Figure 2. A desorption rate of over $90 \%$ was observed for the D-3250, D-140, D-101 and AB-8 resins, using 40\% ethanol, whilst AB-8 exhibited a similar desorption rate with $60 \%$ ethanol. In addition to the NKA-II resin and polyamide, the desorption rate of other resins at $40 \%$ and $70 \%$ ethanol was significantly better than that of $100 \%$ desorption solutions. The desorption rate of NKA-II was also significantly lower compared to other resins. Therefore, comprehensive consideration of the static adsorption and desorption characteristics of different resins HPD-300, HPD-600, D-3250, X-5, D-140, NKA-9, D-101 and AB-8 resins were applied for the following study.

The above results showed that the material, polarity, specific surface area and pore diameter of these resins were important for the adsorption and desorption capacities of EUOL polyphenol. The polarities of different macroporous resins depended on their materials. Generally, non-polar macroporous resins were mainly composed of styrene and divinylbenzene polymers; medium polar macroporous resins were mostly polyacrylate polymers, and multifunctional methacrylates were used as crosslinking agents; the polar macroporous resin mainly contains sulfur and oxygen and amide group; for the strong polar macroporous resin, it mainly contain nitrogen oxide group strongly polar compounds generally needed to be enriched with medium polar resins, and non-polar resins could be used for the separation of weak polar compounds. The pore diameters of the macroporous resin would affect the movement of active compounds in the resin, which would also determine the rate of adsorption and desorption. Resins with higher adsorption and desorption ratios had large specific surface areas, which was another critical factor that affected the adsorption and desorption of polyphenol in EUOL when macroporous resins were used.

\section{Static adsorption kinetic of target compounds}

The adsorption kinetic curves of resins selected are shown in Figure $3 A$. The adsorption capacities reached equilibrium after $80 \mathrm{~min}$. The adsorption processes for all the resins exhibited three stages, including fast-, slow adsorption and equilibrium. At the initial stage, the adsorption capacities of the resins were linear and rapid over the first $15 \mathrm{~min}$. The adsorption of NKA-9, D-3250 and HPD-300 resin were the most swift compared to the other resins. The adsorption capacity rates of all the resins decreased at the second stage, finally achieving equilibrium at $80 \mathrm{~min}$. 

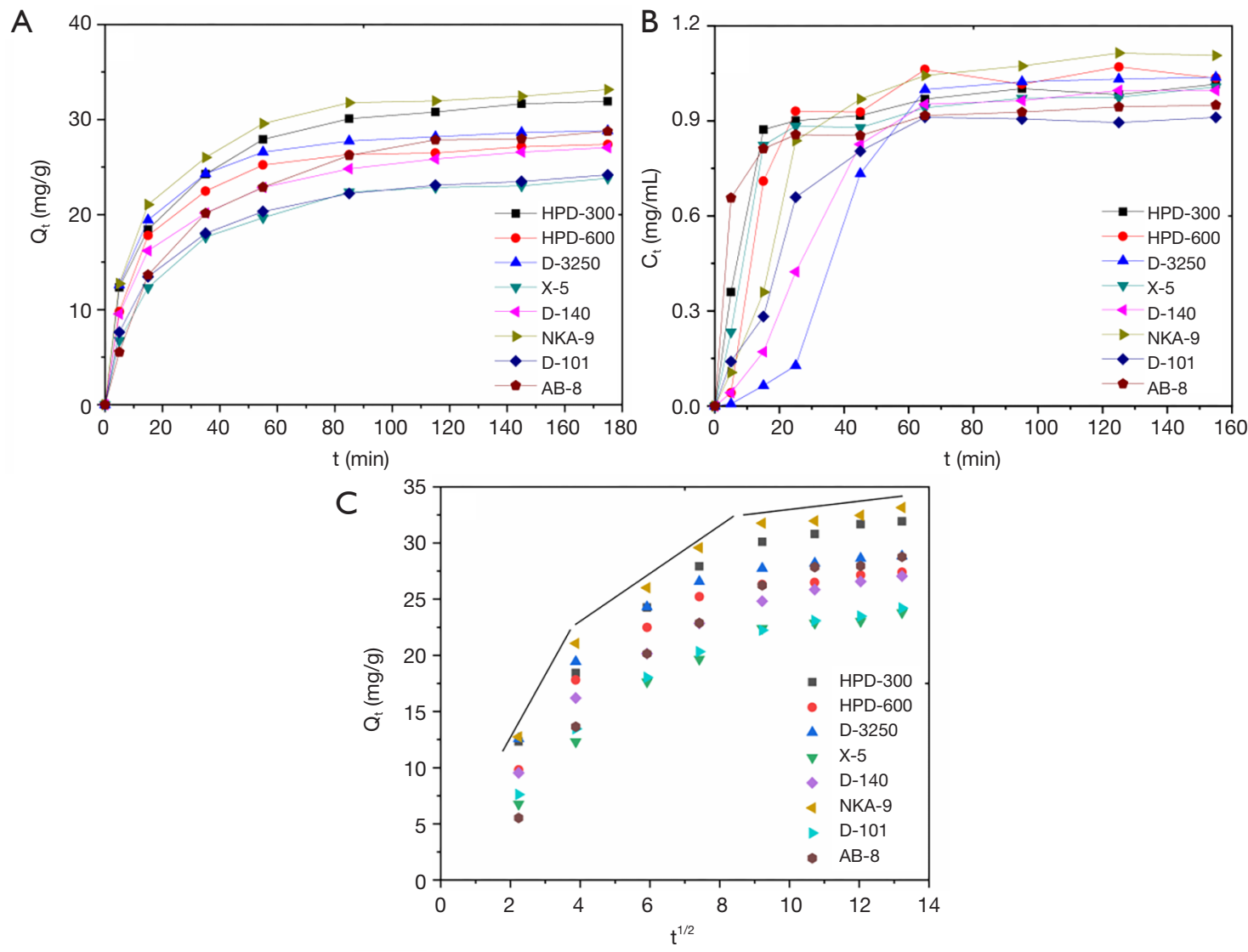

Figure 3 Adsorption and desorption properties of different resins. (A) Adsorption curves, (B) desorption curves, (C) internal particle diffusion.

Whilst the adsorption curves of different resins displayed similar trends, the differences between the adsorption rate and the adsorption amount was notable. Among these resins, the adsorption rate and equilibrium adsorption capacity of X-5 and D-101 were relatively low. Thus, these were considered as rapid adsorption resins. At the same time, the desorption kinetic curves of different resins were also investigated. As shown in the Figure 3B, the difference between the desorption curves of different resins is more pronounced, compared with Figure $3 \mathrm{~A}$. All resins essentially achieved desorption equilibrium after $45 \mathrm{~min}$. D-3250 was shown to exhibit the slowest desorption rate, whilst $\mathrm{AB}-8$ demonstrated the most rapid, achieving desorption within $15 \mathrm{~min}$. The desorption amount of NKA-9 was the highest among all tested resins. The concentration of desorption solution ranged between $0.9-1.1 \mathrm{mg} / \mathrm{mL}$. Based on the adsorption and desorption curves, the appropriate resin can be selected, based on the required adsorption rate or amount.
The adsorption process is widely recognized as an unstable state, and the rate is closely related to the duration, solvent, and type of adsorbent. The adsorption of solutes is generally divided into three stages: (I) mass transfer of adsorbate molecules through the boundary layer, (II) internal particle diffusion in the pores of the adsorbent, (III) adsorption at a site on the adsorbent surface (40). In order to elucidate the adsorption behaviors and mechanisms of all resins, pseudofirst-order, pseudo-second-order and particle diffusion kinetics models were chosen to evaluate the adsorption processes. The pseudo-first-order model is generally applicable over the initial stage of an adsorption process. However the pseudo-second-order model assumes that the rate-limiting step is chemisorption and predicts the behavior over the whole range of adsorption (41). These equations, including derived parameters such as correlation coefficient and dynamic parameters, are summarized in Table 2.

The pseudo-second-order kinetic model were chosen as most favorable for exhibiting the adsorption processes 
Table 2 Equation of pseudo-first-order, pseudo-second-order and particle diffusion kinetics model and dynamic parameters for different resins

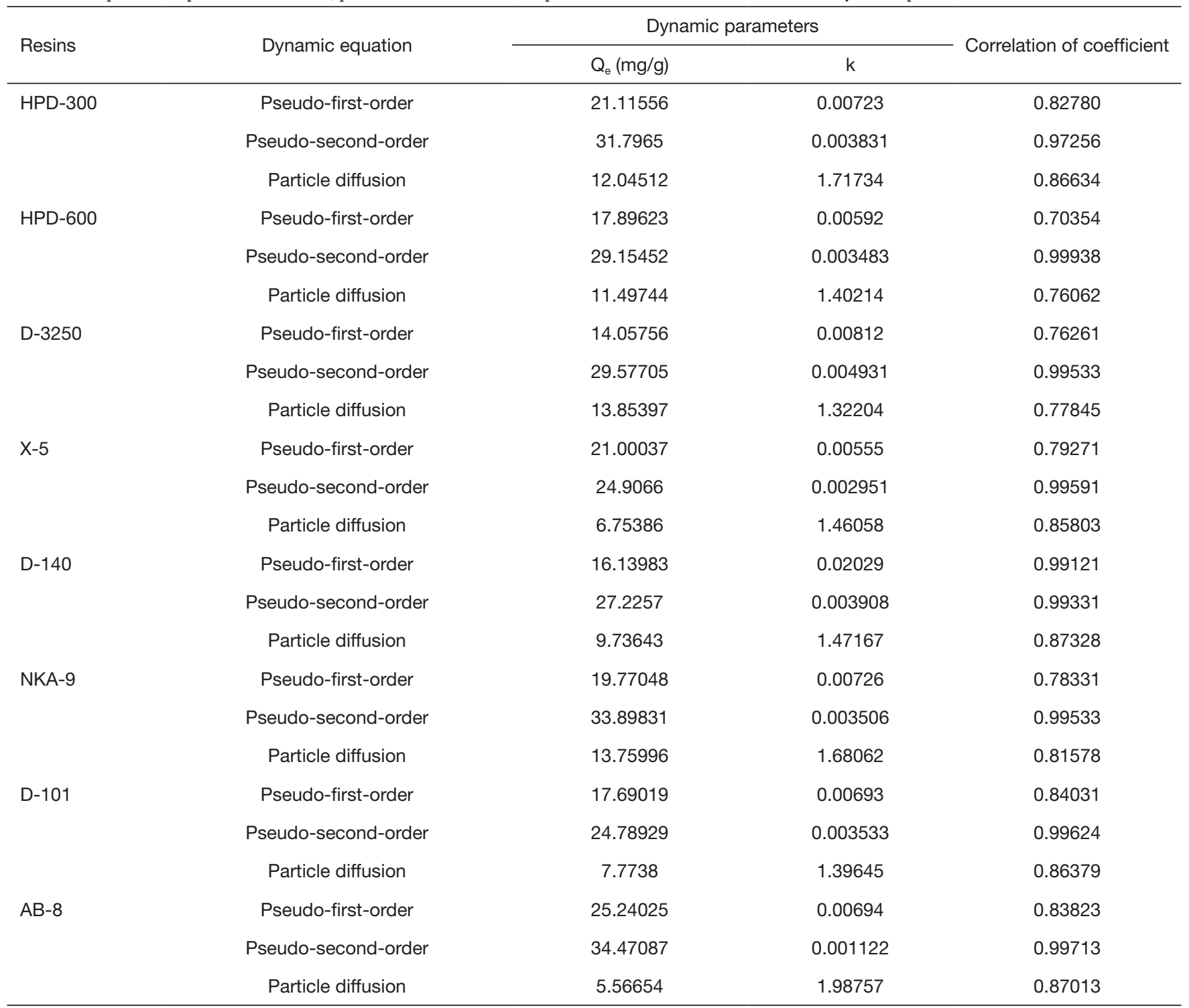

of EUOL polyphenols on these resins, due to the strong correlation obtained (Table 2). At the same time, the calculated values of the adsorption capacity fitted well with the experimental results for every elected resin. This result suggested that the pseudo-second-order kinetic model was fit for evaluating the adsorption capacities of polyphenols in EUOL using these eight resins.

In addition, although the particle diffusion kinetic models could not represent the entire adsorption processes for the weak linear trends, they could describe the adsorption mechanism at one stage of the adsorption process. The diffusion curves of three resins $\left(Q_{t}\right.$ versus $\left.t^{1 / 2}\right)$ were shown in Figure 3C. The plots exhibit weak linear trends over the duration selected. Thus, the whole process was divided into three stages, suggesting that the adsorption of the polyphenol in EUOL on these resins might comprise of multiple processes. The first stage $(0-15 \mathrm{~min})$, pertained to boundary layer diffusion, while the second phase (15-65 min) described the gradual adsorption stage, where intra-particle diffusion was rate-limited. The third stage $(65-175 \mathrm{~min}$ for all resins) represented the final equilibrium stage. The plots did not pass through the origin, indicating that both boundary layer diffusion and intra-particle diffusion were the rate-controlling factors of adsorption (42). Above all, 
Table 3 The parameters of Langmuir and Freundlich models for three resins

\begin{tabular}{|c|c|c|c|c|c|c|c|}
\hline Resins & $\mathrm{T}\left({ }^{\circ} \mathrm{C}\right)$ & \multicolumn{3}{|c|}{ Langmuir } & \multicolumn{3}{|c|}{ Freundlich } \\
\hline \multirow[t]{3}{*}{ HPD-300 } & 25 & 111.9821 & 0.5207 & 0.9799 & 1.1945 & 42.4268 & 0.9946 \\
\hline & 35 & 79.6178 & 0.8710 & 0.9815 & 1.2718 & 36.9612 & 0.9812 \\
\hline & 45 & 35.7654 & 2.8271 & 0.9140 & 1.5488 & 31.1867 & 0.9258 \\
\hline \multirow{2}{*}{ HPD-600 } & 35 & 69.1563 & 0.6757 & 0.9982 & 1.3578 & 31.2347 & 0.9989 \\
\hline & 45 & 48.1928 & 1.3233 & 0.9793 & 1.4074 & 29.0126 & 0.9970 \\
\hline \multirow[t]{2}{*}{ NKA-9 } & 25 & 406.5041 & 0.0935 & 0.9997 & 1.0589 & 34.2744 & 0.9987 \\
\hline & 35 & 72.3066 & 0.6605 & 0.9833 & 1.2108 & 30.2895 & 0.9947 \\
\hline
\end{tabular}

Table 4 Thermodynamic parameters of three resins on the adsorption process

\begin{tabular}{|c|c|c|c|c|}
\hline Resins & $\mathrm{T}(\mathrm{K})$ & $\Delta \mathrm{S}[\mathrm{J} /(\mathrm{mol} \mathrm{K})]$ & $\Delta \mathrm{H}(\mathrm{kJ} / \mathrm{mol})$ & $\Delta \mathrm{G}(\mathrm{kJ} / \mathrm{mol})$ \\
\hline \multirow{2}{*}{ HPD-300 } & 308 & & & -3.6683 \\
\hline & 318 & & & -3.6636 \\
\hline \multirow[t]{2}{*}{ HPD-600 } & 298 & -0.4398 & -3.6848 & -3.5538 \\
\hline & 318 & & & -3.5450 \\
\hline \multirow[t]{3}{*}{ NKA-9 } & 298 & -0.9220 & -3.7061 & -3.4314 \\
\hline & 308 & & & -3.4222 \\
\hline & 318 & & & -3.4129 \\
\hline
\end{tabular}

eight resins exhibited similar adsorption behaviors and possessed good adsorption capacities. Additionally, this result illustrated that the phenolics possessing benzene rings and hydrogen groups could be absorbed by weak polar and non-polar resins with proper particle size, specific surface area and average pore diameter. The $\pi-\pi$ conjugation between phenolic compounds and the benzene rings of these resins may be an important force in the adsorption processes of phenolics on these resins (26).

\section{Adsorption isotherms}

The Langmuir and Freundlich models are often chosen to describe adsorption isotherm due to their simplicity and accuracy (30). Unlike the Langmuir model, the Freundlich isotherm model, which is an empirical model, assumes that the adsorption is multilayer, and the ratio of adsorbed solute to its concentration is a function of the total solute concentration $(43,44)$.

The Langmuir and Freundlich equations were used for curve fitting by Origin 2019b, and the corresponding parameters of the different equations are displayed in Table 3. By comparing the correlation coefficients $\left(R^{2}\right)$, the Freundlich isotherm model best described the adsorption characteristics of polyphenol on these resins, followed by the Langmuir model (Table 3). In addition, the decrease in $\mathrm{Q}_{\mathrm{m}}$ with increasing temperature indicated that high temperature suppressed flavonoid adsorption, consistent with the results from previous reports (45). For Freundlich model, the constant $\mathrm{n}$ can be used to explain the adsorption isotherm type. The adsorption is 
Table 5 Total polyphenol content and enzyme inhibitory activity of the fraction eluted from column packed with HPD-300 resin

\begin{tabular}{lccc}
\hline \multirow{2}{*}{ Ethanol concentration } & Content of polyphenol $(\mathrm{mg} / \mathrm{g})$ & \multicolumn{2}{c}{$\mathrm{IC}_{50}(\mathrm{mg} / \mathrm{mL})$} \\
\cline { 3 - 4 } & $8.6451 \pm 0.0193$ & $\alpha$-Amylase inhibition & $\alpha$-Glucosidase inhibition \\
\hline Water & $60.7134 \pm 1.2209$ & $10.27 \pm 0.3385$ & $5.98 \pm 0.1874$ \\
$20 \%$ ethanol & $1,084.0614 \pm 2.1384$ & $6.71 \pm 0.1361$ & $1.93 \pm 0.2103$ \\
$40 \%$ ethanol & $1,680.3304 \pm 2.0861$ & $2.05 \pm 0.0287$ & $1.15 \pm 0.1010$ \\
$60 \%$ ethanol & $1,004.3971 \pm 0.3390$ & $3.54 \pm 0.2984$ & $3.09 \pm 0.0090$ \\
$80 \%$ ethanol & $32.5891 \pm 1.0296$ & $5.31 \pm 0.2827$ & $5.17 \pm 0.1123$ \\
$100 \%$ ethanol & $152.4123 \pm 0.5989$ & $8.44 \pm 0.1898$ & $8.21 \pm 0.0381$ \\
Crude extract & - & $0.136 \pm 0.0019$ & $0.098 \pm 0.002$ \\
Acarbose & & & \\
\hline
\end{tabular}

favorable when $0<1 / \mathrm{n}<1$ and unfavorable when $1 / \mathrm{n}>1$ (46). The results obtained from this study showed that the adsorption process was favorable because $\mathrm{n}>1$.

\section{Adsorption thermodynamics of target compounds on the selected resins}

Adsorption thermodynamics can be used to further reflect changes in the internal structure and energy of the adsorption process. A plot of $\ln K_{p}$ against $1 / T$ yielded a straight line. The $\Delta G$ and $\Delta S$ values obtained from the slope and intercept of the plot are listed in Table 4. Essentially, the heat generated during physical adsorption is of the same order of level as the heat of condensation, i.e., $2.1-20.9 \mathrm{~kJ} / \mathrm{mol}$, while the heat of chemical adsorption is in the range of $80-200 \mathrm{~kJ} / \mathrm{mol}$ (47). The absolute value of $\Delta \mathrm{H}$ was lower than $20 \mathrm{~kJ} / \mathrm{mol}$, and showed that the adsorption was attributed to physical adsorption, rather than chemisorption. Furthermore, negative $\Delta \mathrm{G}$ and $\Delta \mathrm{H}$ values were obtained for the adsorption of polyphenol on the resins surfaces, indicating that the adsorption was exothermic and spontaneous. The $\Delta \mathrm{G}$ increased with temperature from 298 to $318 \mathrm{~K}$, which illustrated that adsorption was favored at lower temperatures $(29,48)$.

\section{Analyses of polyphenol from different fraction eluted by column chromatography}

Based on the above experimental results, HPD-300 was selected for dynamic column chromatography. The polyphenol in EUOL was dynamically enriched and eluted with ethanol solutions of different concentrations. The polyphenol content in different eluents from the selected resins is shown in the Table 5. The content of polyphenols in $40 \%$ and $80 \%$ ethanol was similar, and both were at higher levels than the crude extract. The highest content of polyphenol $(1,680.3304 \mathrm{mg} / \mathrm{g})$ was observed in sample solutions eluted with $60 \%$ aqueous ethanol, which was eight times that of the crude extract. About $95 \%$ of polyphenol were presented in $40 \%, 60 \%$ and $80 \%$ ethanol fractions. The total polyphenol content in the water eluent and $100 \%$ ethanol elution solution was lower than the original crude sample solution. The original concentration of the target compounds in the EUOLs were $152.4123 \mathrm{mg} / \mathrm{g}$. After treatment with the selected resin, the polyphenol concentration substantially increased by approximately eleven times. This may be due to the scarcity of relatively large or small polar polyphenols in the extract sample, with the majority of polyphenols found to be of medium polarity. HPD-300 might be used as an effective resin for enrichment of polyphenol in EUOL.

The eluates obtained from the above column chromatography were assessed for their inhibitory effect on $\alpha$-amylase and $\alpha$-glucosidase activity. The results were presented in the Table 5, and indicated that the eluates have inhibitory activity on $\alpha$-amylase and $\alpha$-glucosidase activity. Furthermore, the calculation result of $\mathrm{IC}_{50}$ value showed that high inhibitory activity were found to be in fractions eluted with $40 \%, 60 \%$ and $80 \%$ ethanol. The inhibitory effect of $60 \%$ ethanol was the most pronounced, although it was less than that of the positive control group. This result may be attributed to the complex composition of the sample components, and these compounds had an excellent inhibitory effect on $\alpha$-amylase and $\alpha$-glucosidase activity. Components 
A

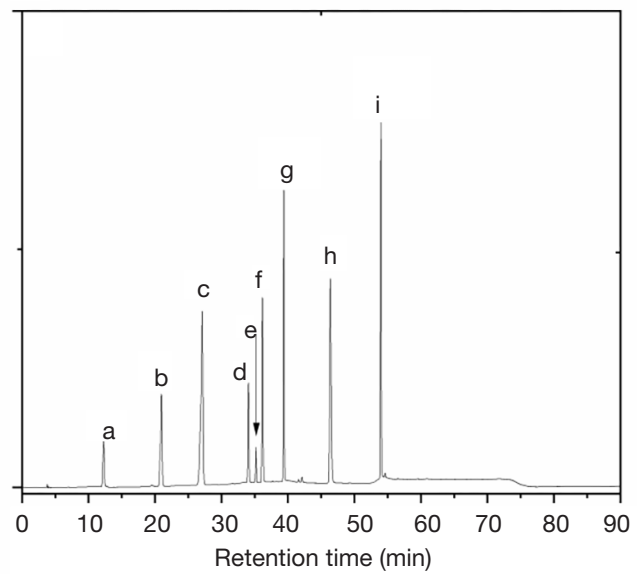

B

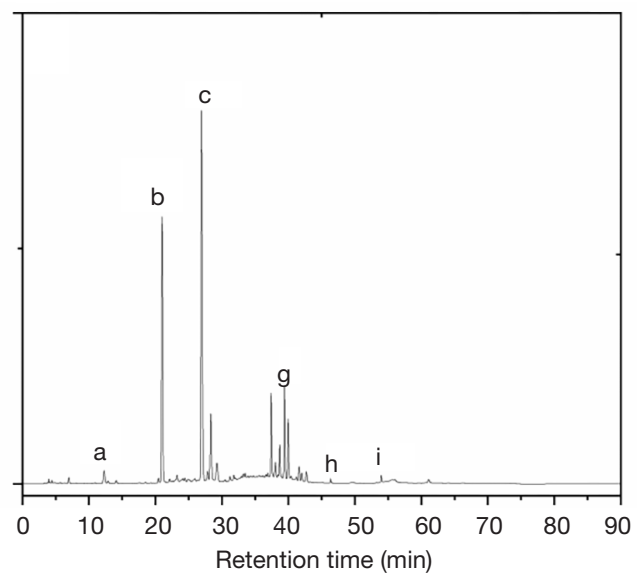

Figure 4 HPLC chromatograms of reference materials (A) and samples (B). a, aucubin; b, geniposide; c, chlorogenic acid; d, geniposide; e, pinoresinol diglucoside; f, genipin; g, rutin; h, quercetin; i, kaempferol. HPLC, high-phase liquid chromatography.

Table 6 Information on the main polyphenol compounds and their molecular docking results

\begin{tabular}{lcccc}
\hline \multirow{2}{*}{ Compounds } & Molecular formula & Molecular weight, g/moL & \multicolumn{2}{c}{ Total score } \\
\cline { 3 - 5 } Chlorogenic acid & $\mathrm{C}_{16} \mathrm{H}_{18} \mathrm{O}_{9}$ & 354.3 & $\alpha$-Amylase & 2.7140 \\
Rutin & $\mathrm{C}_{27} \mathrm{H}_{30} \mathrm{O}_{16}$ & 610.5 & 4.3419 & 3.9591 \\
Quercetin & $\mathrm{C}_{15} \mathrm{H}_{10} \mathrm{O}_{7}$ & 302.2 & 4.0134 & 5.3419 \\
Kaempferol & $\mathrm{C}_{15} \mathrm{H}_{10} \mathrm{O}_{6}$ & 286.2 & 2.5705 & 2.7611 \\
\hline
\end{tabular}

with inhibition effect on enzymes and high polyphenol content can be used for the development of antioxidants, nutritional supplements, or pharmaceutical therapies. Therefore, the composition of this fraction was analyzed for quantitation by HPLC. And the chromatogram was showed in Figure 4. Chlorogenic acid, rutin, quercetin and kaempferol were found to be the predominant polyphenols.

Furthermore, the SYBYL X 2.0 molecular docking software was used to simulate the protein interactions with four small polyphenols. The docking scores are presented in Table 6 . The various compounds displayed different scores for the degree of docking with $\alpha$-amylase and $\alpha$-glucosidase. Rutin displayed the highest docking score with $\alpha$-amylase, followed by quercetin, chlorogenic acid, and kaempferol. However, quercetin exhibited a higher docking score for $\alpha$-glucosidase, compared to rutin. The degree of kaempferol binding was relatively weak among these compounds Figure 5. Figure 5 A,B illustrate the docking results of rutin and quercetin with amylase, respectively. The docking results of glucosidase and the target compound (quercetin and rutin) are shown in Figure 5C,D. To achieve the best binding mode between the compound and the enzyme, each compound could dock with the macromolecular in many different conformation, and the molecular conformation with higher docking score was considered to be the best optimized state. Due to the differences in the active sites of the enzymes, the binding methods of the same compounds would differ. Similarly, for the same active site, the molecular size, structure, and conformation of the target compound would also affect its affinity with the enzyme. The results of this study indicate that quercetin and rutin were more suitable active sites for these two enzymes. This may provide some theoretical reference for further explaining the results of the above enzyme activity inhibition test.

\section{Discussion}

EUO is an important plant resource and belongs to precious 

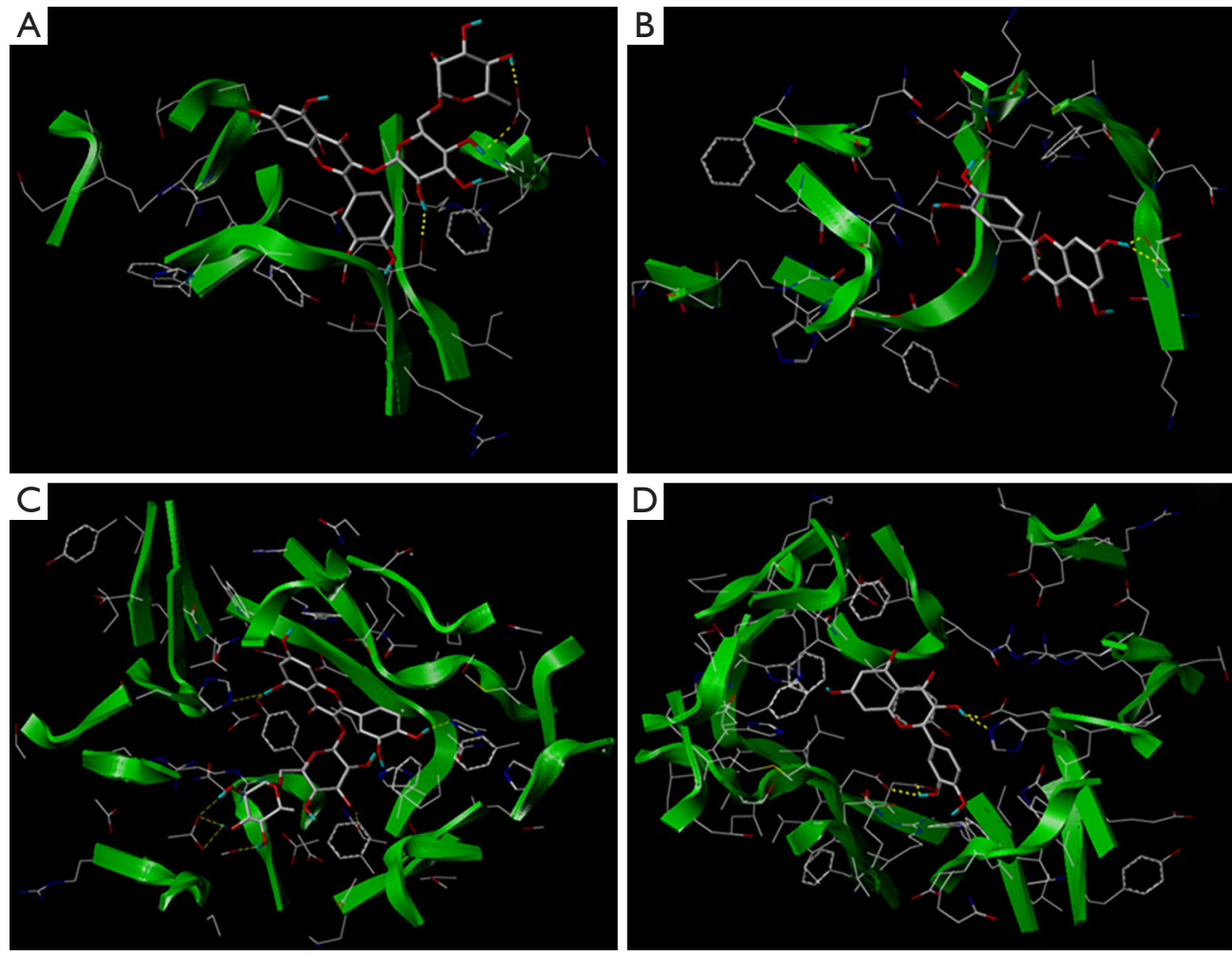

Figure 5 Molecular docking results of different compounds with $\alpha$-amylase (A,B) and $\alpha$-glucosidase (C,D).

traditional Chinese medicinal materials. At present, 132 compounds have been obtained from bark, leaves, flowers and seeds of EUO. Among them, EUOL has attracted attention due to its easy collection, short growth cycle and high application value. There have been reports on the enrichment, separation and purification of active ingredients in EUOL, but there are few reports on the systematic study of the enrichment of polyphenols as the main type of compound in EUOL. At the same time, current studies have found polyphenols substances as dietary additives can effectively delay diabetes. Therefore, based on the systematic investigation of the adsorption and desorption characteristics of polyphenols, this study screened enzyme inhibitors based on the active sites of polyphenols, and obtained interesting results. However, the characteristic component composition or synergistic effect of the enzyme inhibitory effect on the active site still needs to be further studied.

The purpose of the preparation of active ingredients and the study of biological activity is to achieve highvalue utilization of resources. Active substances are also the material basis for biological effects. EUOL resources are very abundant in our country. In order to strengthen the deep processing and utilization of EUOL resources, we should simplify the preparation process of active ingredients, expand the scope of biological activity investigation, accurately screen the target active fractions and clarify the material basis, and ensure the safety and effectiveness of the composition of EUOL. Meanwhile, the source of raw materials should also be strictly controlled to ensure product quality.

\section{Conclusions}

In summary, a simple, environmentally sustainable and efficient approach for the enrichment polyphenols compounds from EUOL was established. Twelve resins were screened for superior adsorption and desorption performance. The static adsorption data including kinetics and isotherms were analyzed with empirical equations. It was found that the kinetic data could better fit the pseudosecond-order kinetics model, and the isotherms were appropriately described by Freundlich isotherm models. These results indicated that the adsorption mechanism was multimolecular layer adsorption. The parameters $(\Delta G$, 
$\Delta \mathrm{H}$ and $\Delta \mathrm{S}$ ) of adsorption thermodynamics indicated that this process was spontaneous, physical, and exothermic. Dynamic elution results showed polyphenols of EUOL was enriched in $40 \%, 60 \%$ and $80 \%$ ethanol fractions. Additionally, this method significantly increased the total polyphenol content from $152.4123 \%$ to $1,680.3304 \%$. Furthermore, chlorogenic acid, rutin, quercetin and kaempferol were considered the main polyphenolic compounds by HPLC. The results of molecular docking suggested that rutin and quercetin played an important role in the inhibition of $\alpha$-amylase and $\alpha$-glucosidase. Therefore, the macroporous resin enrichment established in this study is a sustainable technique for enrichment of polyphenol from EUOL without using toxic solvents. This method, therefore, has wide applications in food and pharmaceutical studies, with a manufacturing potential at an industrial scale.

\section{Acknowledgments}

Funding: This work was supported by the GDAS' Project of Science and Technology Development (2019GDASYL-0103024, 2019GDASYL-0502003), the National Natural Science Foundation of China (31660181), and project funded by the China Postdoctoral Science Foundation (2019M653099), the Key Laboratory of Human Forest Products and Chemical Industry Engineering (LCHG1902), the Educational Commission of Hunan Province of China (19C1532).

\section{Footnote}

Reporting Checklist: The authors have completed the MDAR reporting checklist. Available at http://dx.doi.org/10.21037/ atm-20-5468

Data Sharing Statement: Available at http://dx.doi. org/10.21037/atm-20-5468

Conflicts of Interest: All authors have completed the ICMJE uniform disclosure form (available at http://dx.doi. org/10.21037/atm-20-5468). The authors have no conflicts of interest to declare.

Ethical Statement: The authors are accountable for all aspects of the work in ensuring that questions related to the accuracy or integrity of any part of the work are appropriately investigated and resolved.
Open Access Statement: This is an Open Access article distributed in accordance with the Creative Commons Attribution-NonCommercial-NoDerivs 4.0 International License (CC BY-NC-ND 4.0), which permits the noncommercial replication and distribution of the article with the strict proviso that no changes or edits are made and the original work is properly cited (including links to both the formal publication through the relevant DOI and the license). See: https://creativecommons.org/licenses/by-nc-nd/4.0/.

\section{References}

1. Zhu MQ, Sun RC. Eucommia ulmoides Oliver: A Potential Feedstock for Bioactive Products. J Agric Food Chem 2018;66:5433-8.

2. Hirata T, Ikeda T, Fujikawa T, et al. The Chemistry and Bioactivity of Eucommia ulmoides Oliver Leaves. Stud Nat Prod Chem 2014;41:225-60.

3. Cheung HY, Lai WP, Cheung MS, et al. Rapid and simultaneous analysis of some bioactive components in Eucommia ulmoides by capillary electrophoresis. J Chromatogr A 2003;989:303-10.

4. National Commission of Chinese Pharmacopoeia. Pharmacopoeia of the People's Republic of China (Vol. 1). Beijing: China Medical Science and Technology Press, 2015:154.

5. He X, Wang J, Li M, et al. Eucommia ulmoides Oliv.: ethnopharmacology, phytochemistry and pharmacology of an important traditional Chinese medicine. J Ethnopharmacol 2014;151:78-92.

6. Zhou Y, Liang M, Li W, et al. Protective effects of Eucommia ulmoides Oliv. bark and leaf on amyloid $\beta$-induced cytotoxicity. Environ Toxicol Pharmacol 2009;28:342-9.

7. Hsieh CL, Yen GC. Antioxidant actions of Du-Zhong (Eucommia ulmoides Oliv.) toward oxidative damage in biomolecules. Life Sci 2000;66:1387-400.

8. Xiang Y, Huang RH, Liu XZ, et al. Crystal structure of a novel antigungal protein distinct with five disulfide bridges from Eucommia ulmoides Oliver at an atomic resolution. J Struct Biol 2004;148:86-97.

9. Kwan CY, Chen CX, Deyama T, et al. Endotheliumdependent vasorelaxant effects of the aqueous extracts of the Eucommia ulmoides Oliv. leaf and bark: implications on their antihypertensive action. Vascul Pharmacol 2003;40:229-35.

10. Lee GW, Yoon HC, Byun SY. Inhibitory effect of Eucommia ulmoides Oliver on adipogenic differentiation 
through proteome analysis. Enzyme Microb Tech 2004;35:632-8.

11. Yen GC, Hsieh CL. Inhibitory effects of Du-zhong (Eucommia ulmoides Oliv.) against low-density lipoprotein oxidative modification. Food Chem 2002;77:449-56.

12. Nakamura T, Nakazawa Y, Onizuka S, et al. Antimutagenicity of Tochu tea (an aqueous extract of Eucommia ulmoides leaves): 1. The clastogen-suppressing effects of Tochu tea in CHO cells and mice. Mutat Res 1997;388:7-20.

13. Kim HY, Moon BH, Lee HJ, et al. Flavonol glycosides from the leaves of Eucommia ulmoides Oliv. with glycation inhibitory activity. J Ethnopharmacol 2004;93:227-30.

14. Lee MK, Kim MJ, Cho SY, et al. Hypoglycemic effect of Du-zhong (Eucommia ulmoides Oliv.) leaves in streptozotocin-induced diabetic rats. Diabetes Res Clin Pract 2005;67:22-8.

15. Park SA, Choi MS, Kim MJ, et al. Hypoglycemic and hypolipidemic action of Du-zhong (Eucommia ulmoides Oliver) leaves water extract in C57BL/KsJ-db/db mice. J Ethnopharmacol 2006;107:412-7.

16. Yang J, Kato K, Noguchi K, et al. Tochu (Eucommia ulmoides) leaf extract prevents ammonia and vitamin $\mathrm{C}$ deficiency induced gastric mucosal injury. Life Sci 2003;73:3245-56.

17. Bahadoran Z, Mirmiran P, Azizi F. Dietary polyphenols as potential nutraceuticals in management of diabetes: a review. J Diabetes Metab Disord 2013;12:43.

18. Annunziata G, Jiménez-García M, CapóX, et al. Microencapsulation as a tool to counteract the typical low bioavailability of polyphenols in the management of diabetes. Food Chem Toxicol 2020;139:111248.

19. Wang J, Wu T, Fang L, et al. Anti-diabetic effect by walnut (Juglans mandshurica Maxim.)-derived peptide LPLLR through inhibiting $\alpha$-glucosidase and $\alpha$-amylase, and alleviating insulin resistance of hepatic HepG2 cells. J Funct Foods 2020;69:103944.

20. Kawde AN, Taha M, Alansari RS, et al. Exploring efficacy of indole-based dual inhibitors for $\alpha$-glucosidase and $\alpha$-amylase enzymes: In silico, biochemical and kinetic studies. Int J Biol Macromol 2020;154:217-32.

21. Zhong JL, Muhammad N, Gu YC, et al. A simple and efficient method for enrichment of cocoa polyphenols from cocoa bean husks with macroporous resins following a scale-up separation. J Food Eng 2019;243:82-8.

22. Jia $G, L u X$. Enrichment and purification of madecassoside and asiaticoside from Centella asiaticaextracts with macroporous resins. J Chromatogr A 2008;1193:136-41.
23. Hashimoto T. Macroporous synthetic hydrophilic resinbased packings for the separation of biopolymers. J Chromatogr A 1991;544:249-55.

24. Jin X, Liu MY, Chen ZX, et al. Separation and purification of epigallocatechin-3-gallate (EGCG) from green tea using combined macroporous resin and polyamide column chromatography. J Chromatogr B Analyt Technol Biomed Life Sci 2015;1002:113-22.

25. Lv C, Yang J, Liu R, et al. A comparative study on the adsorption and desorption characteristics of flavonoids from honey by six resins. Food Chem 2018;268:424-30.

26. Yang Q, Zhao M, Lin L. Adsorption and desorption characteristics of adlay bran free phenolics on macroporous resins. Food Chem 2016;194:900-7.

27. Wu SH, Wang YY, Gong GL, et al. Adsorption and desorption properties of macroporous resins for flavonoids from the extract of Chinese wolfberry (Lycium barbarum L.). Food Bioprod Process 2015;93:148-55.

28. Xiong N, Yu R, Chen T, et al. Separation and purification of l-methionine from E. coli fermentation broth by macroporous resin chromatography. J Chromatogr B Analyt Technol Biomed Life Sci 2019;1110-1111:108-15.

29. Liu C, Jiao R, Yao L, et al. Adsorption characteristics and preparative separation of chaetominine from Aspergillus fumigatus mycelia by macroporous resin. J Chromatogr B Analyt Technol Biomed Life Sci 2016;1015-1016:135-41.

30. Yuanfeng $W$, Lei Z, Jianwei $M$, et al. Kinetic and thermodynamic studies of sulforaphane adsorption on macroporous resin. J Chromatogr B Analyt Technol Biomed Life Sci 2016;1028:231-6.

31. Wang L, Boussetta N, Lebovka N, et al. Ultrasound assisted purification of polyphenols of apple skins by adsorption/desorption procedure. Ultrason Sonochem 2019;55:18-24.

32. Wu D, Yu DM, Zhang YJ, et al. Metabolite profiles, bioactivity, and HPLC fingerprint of different varieties of Eucommia ulmoides Oliv.: towards the utilization of medicinal and commercial Chinese endemic tree. Molecules 2018;23:1898.

33. Cardullo N, Muccillia V, Pulvirenti L, et al. C-glucosidic ellagitannins and galloylated glucoses as potential functional food ingredients with anti-diabetic properties: a study of $\alpha$-glucosidase and $\alpha$-amylase inhibition. Food Chem 2020;313,126099.

34. Thengyai S, Thiantongin $\mathrm{P}$, Sontimuang $\mathrm{C}$, et al. $\alpha$-Glucosidase and $\alpha$-amylase inhibitory activities of medicinal plants in Thai antidiabetic recipes and bioactive compounds from Vitex glabrata R. Br. stem bark. J Herb 
Med 2020;19:100302.

35. Sandhu AK, Gu L. Adsorption/desorption characteristics and separation of anthocyanins from muscadine (Vitis rotundifolia) juice pomace by use of macroporous adsorbent resins. J Agric Food Chem 2013;61:1441-8.

36. Deosarkar S, Pangarkar V. Adsorptive separation and recovery of organics from PHBA and SA plant effluents. Sep Purif Technol 2004;38:241-54.

37. Lin L, Zhao H, Dong Y, et al. Macroporous resin purification behavior of phenolics and rosmarinic acid from Rabdosia serra (MAXIM.) HARA leaf. Food Chem 2012;130:417-24.

38. Amin LP, Pangarkar VG, Beenackers A. Recovery of valuable perfumery compounds from a geranium steam distillation condensate using polymeric adsorbents. Sep Sci Technol 2001;36:3639-55.

39. Cai Y, Lu XH, Wei QL. Studies of isolation for total flavonoids in Rhodobryum giganteum by polyamide column adsorption chromatogtaphy. J Pharm Practice 2009;27:58-60.

40. Liu QS, Zheng T, Wang P, et al. Adsorption isotherm, kinetic and mechanism studies of some substituted phenols on activated carbonfibers. Chem Eng J 2010;157:348-56.

41. Duran C, Ozdes D, Gundogdu A, et al. Kinetics and isotherm analysis of basic dyes adsorption onto Almond Shell (Prunus dulcis) as a low cost adsorbent. J Chem Eng Data 2011;56:2136-47.

42. Lorenc-Grabowska E, Gryglewicz G. Adsorption of

Cite this article as: Wang Z, Peng S, Peng M, She Z, Yang Q, Huang T. Adsorption and desorption characteristics of polyphenols from Eucommia ulmoides Oliv. leaves with macroporous resin and its inhibitory effect on $\alpha$-amylase and $\alpha$-glucosidase. Ann Transl Med 2020;8(16):1004. doi: 10.21037/ atm-20-5468 lignite-derived humic acids on coal-based mesoporous activated carbons. J Colloid Interface Sci 2005;284:416-23.

43. Gao ZP, Yu ZF, Yue TL, et al. Adsorption isotherm: thermodynamics and kinetics studies of polyphenols separation from kiwifruit juice using adsorbent resin. J Food Eng 2013;116:195-201.

44. Rajabi M, Barfi B, Asghari A, et al. Hybrid aminefunctionalized titania/silica nanoparticles for solid-phase extraction of lead, copper, and zinc from food and water samples: kinetics and equilibrium studies. Food Anal Method 2015;8:815-24.

45. Li C, Liang H, Yuan Q, et al. Optimization of sulforaphane separation from broccoli seeds by macroporous resins. Sep Sci Technol 2008;43:609-23.

46. Vasiliu S, Bunia I, Racovita S, et al. Adsorption of cefotaxime sodium salt on polymer coated ion exchange resin microparticles: kinetics, equilibrium and thermodynamic studies. Carbohyd Polym 2011;85:376-87.

47. Liu Y, Liu Y. J. Biosorption isotherms: kinetics and thermodynamics. Sep Purif Technol 2008;61:229-42.

48. Zou SP, Liu M, Wang QL, et al. Preparative separation of echinocandin B from Aspergillus nidulans broth using macroporous resin adsorption chromatography. J Chromatogr B Analyt Technol Biomed Life Sci 2015;978979:111-7.

(English Language Editor: E. Tan) 